\title{
Universalisierte Opferschaft. Über den Erfolg des islamischen Antisemitismus in Europa
}

\author{
Soma Mohammad Assad
}

Die Proteste gegen Israels „Operation Protective Edge“ im Sommer 2014 stellten einen Wendepunkt dar: sie zeigten, welche Massenbasis der offene Antisemitismus in Europa nach 1945 wieder errungen hatte. Das Kantor Center der Universität Tel Aviv, das den weltweiten Antisemitismus, der sich in Attacken gegen Juden und gegen jüdische Einrichtungen äußert, dokumentiert, stellte für das zweite Halbjahr 2014 einen rasanten Anstieg in Westeuropa und Nordamerika fest. ${ }^{1}$ Allein bezüglich der „,major cases“ stieg in Frankreich die Zahl der verbalen und tätlichen Übergriffe von 423 auf 851, in Deutschland von 788 auf 1076 und in Österreich von 137 auf 255 Vorfälle. In Großbritannien hatte sich im Vergleich zum Vorjahr die Zahl der gemeldeten Vorfälle sogar mehr als verdoppelt: von 535 im Jahr 2013 auf 1168. ${ }^{2}$ Auch wenn im Jahr 2013 und im ersten Halbjahr 2014 die Zahl bereits erschreckend hoch war, sehen die Studienautoren die Anti-Israel-Demonstrationen gegen den Gaza-Krieg im Sommer 2014 als einen wesentlichen Grund für den starken Anstieg.

„Kindermörder Israel“-Rufe und relativierende Gleichsetzungen Israels mit dem Nationalsozialismus wurden bereits vor den Pro-Gaza-Demonstrationen vermehrt skandiert, jedoch ist mit den Ereignissen im Sommer 2014 das antisemitische Tabu, das angesichts der Agitation antiimperialistischer Gruppierungen und rechtspopulistischer Organisationen und Parteien ohnehin ein oberflächliches war, damals unter großer Mitwirkung von Muslimen durchbrochen worden. Europaweit kam es im Sommer 2014 zu Angriffen auf Synagogen und Übergriffen auf Einzelpersonen, darunter auch Juden. Es herrschte wieder Mob-Stimmung gegen Juden auf den europäischen Straßen. Angesichts dessen ist es auffällig, dass weder die Verbrechen des sogenannten Islamischen Staats, dessen Opfer größtenteils Muslime sind und zu denen unter anderem auch der Angriff auf das palästinensische Flüchtlingslager Yarmouk in Syrien ${ }^{3}$ gehört, noch sonst andere Konflikte im Nahen Osten eine derart große Anzahl an Muslimen auf die europäischen Straßen gebracht hat wie die militärische Selbstverteidigung Israels.

In Gelsenkirchen in Nordrhein-Westfalen wurde „Hamas, Hamas, Juden ins Gas!“ skandiert. ${ }^{4}$ In Frankfurt eskalierte die Situation, als Polizisten gegen Ende der

${ }^{1}$ Kantor Center, Antisemitism Worldwide 2014, o. D.,

[http://mfa.gov.il/mfa/foreignpolicy/antisemitism/documents/kantorreportantisemitism2014.pdf], S. 5, eingesehen 9.11.2018.

2 Ebd., S. 6.

${ }^{3}$ Die Presse, 9.4.2015, S. 4.

${ }^{4}$ Die erwähnten antisemitischen Vorfälle und Übergriffe sind exemplarisch. Für eine weltweite Chronik siehe die 
Demonstration von jungen Männern mit Steinen beworfen und getreten wurden. Als die Demonstration bereits offiziell zu Ende war, formierte sich ein spontaner Protestzug und die Polizei überließ, nach Eigendarstellung, um deeskalierend $\mathrm{zu}$ wirken, einem Demonstranten den Lautsprecherwagen. ${ }^{5}$ Dieser durfte dann mit dem Polizeilautsprecher die Menge unter der Parole „Kindermörder Israel“ führen. In Berlin skandierten überwiegend junge muslimische Männer neben „Allahu Akbar!“ auch „Jude, Jude, feiges Schwein, komm heraus und kämpf allein!“. 6

\section{Das antisemitische Motiv jüdischer Allmacht}

Besonders anhand dieser und ähnlicher Parolen wird das dahinterliegende Motiv einer phantasierten jüdischen Allmacht deutlich, der hier mit Hilfe Allahs der Kampf angesagt wird. Zwar ist jede Religion politisch, jedoch zeigt die erwähnte Kampfansage, dass diese Religion in rein politischer Form und allein als politisches Engagement ihre einzige Gültigkeit haben soll. Der Erfolg der islamischen Ideologie in Europa begründet sich aus einem generellen Ungerechtigkeitsempfinden und einer Opferinszenierung, die - einmal mehr, einmal weniger offenkundig - die USA, den Westen und vor allem Israel als Übeltäter und Verursacher ausmacht. In den westeuropäischen Gesellschaften ist immer mehr zu erkennen, dass auch vonseiten progressiver Kräfte „den“ Muslimen eine entsprechende Opferrolle zugeschrieben wird.

In der erwähnten antisemitischen Parole wird das Stereotyp einer imaginierten jüdischen Allmacht erkennbar: über den Kafir (Ungläubigen), der in der islamischen Ideologie die Stellung des Gegners hat, wird es auf den Juden übertragen und letztendlich in ihm personifiziert. Der gläubige Moslem begeht nach den ideologischen Maßstäben eine Sünde, wenn er das Judentum nicht als monotheistische Religion akzeptiert, doch in dieser Feinderklärung wird der Mechanismus des Antisemitismus erkennbar: Er ist kein „gewöhnliches“ Vorurteil, strukturell liegt sein Unterschied zum Rassismus in der Vehemenz des Irrationalismus. Beides tritt bei autoritären Persönlichkeitsstrukturen auf, aber im antisemitischen Wahn wird der Jude zum absoluten Feind erklärt. Der Antisemitismus hat daher eine gesellschaftsaufhebende Intention. Antisemitismus müsste demzufolge mit mehr Ratio zu verhindern sein, doch gerade mit der Aufklärung im geschichtsphilosophischen Sinne hat sich die universelle Vernunft ihren eigenen „ideologischen Reflex“ geschaffen. Theodor W. Adorno und Max Horkheimer betonen in der „Dialektik der Aufklärung“, dass in der bürgerlichen Gesellschaft der

Reports des Kantor Centers. (Kantor Center, Antisemitism Worldwide, S. 53.)

5 Katharina Iskander/Hans Riebsamen, Anti-Israel-Parolen über Polizeilautsprecher verbreitet, 14.7.2014, [http://www.faz.net/aktuell/rhein-main/demonstration-eskaliert-anti-israel-parolen-ueber-polizeilautsprecherverbreitet-13044034.html], eingesehen 9.11.2018.

${ }^{6}$ Berlin, in: YouTube, 18.7.2014 [https://youtu.be/o4TWI1i4_Nk], eingesehen 9.11.2018. 
Antisemitismus einen „spezifischen ökonomischen Grund“ hat: „die Verkleidung der Herrschaft in Produktion".?

In der bürgerlichen Gesellschaft wurden Freiheit und Gleichheit zwar verankert, jedoch wurde die Herrschaft nicht abgeschafft, sondern durch ein universell geltendes Kapitalverhältnis vermittelt. Ihre Basis ist somit nicht Individualität im emphatischen Sinne, sondern ihre Nivellierung zum Zwecke der Produktion aus der nun eine zugerichtete Form von Individualität hervorgeht: das bürgerliche Subjekt. ${ }^{8}$ Ausbeutung und Herrschaft wurden vereinheitlicht und über den staatlichen Souverän vermittelt. Die nach der Verwertungslogik des Kapitals arbeitende Gesellschaft garantiert dem bürgerlichen Subjekt zwar formal Freiheit und Gleichheit, produziert aber, weil das Versprechen nicht eingelöst wird, gleichsam das Gefühl, betrogen worden zu sein. Hieraus resultiert eine Entfremdung des Subjekts zur Gesellschaft, deren Herrschaftscharakter zugleich verschleiert ist.

Aus dem Gefühl betrogen zu sein resultiert das Bedürfnis dieses Verhältnis aufzuheben, doch artikuliert es sich allzu häufig in Form antisemitischer Ranküne und der Konkretisierung einer personifizierenden Herrschaftskritik. Über den Mechanismus der antisemitischen Projektion, die Adorno und Horkheimer als „falsche Projektion“9 bezeichnen, schreiben sie weiter: „Regungen, die vom Subjekt als dessen eigene nicht durchgelassen werden und ihm doch eigen sind, werden dem Objekt zugeschrieben: dem prospektiven Opfer." ${ }^{10}$ Dabei fühlen sich Antisemiten stets selbst als Opfer und schaffen sich damit ihre eigene Rechtfertigung, ${ }^{11}$ um gegen den bereits als Feind wahrgenommenen Juden vorzugehen: „Die Störung liegt in der mangelnden Unterscheidung des Subjekts zwischen dem eigenen und fremden Anteil am projizierten Material.“12

Die negative Aufhebung des Kapitalverhältnisses hat daher immer einen antisemitischen Kern. Dieser ist nicht spezifisch islamisch, jedoch wird im Islam aufgrund der kollektivistischen Gesellschaftskonstitution jene „Störung“, also schlussendlich das Gefühl, Objekt von anonymen Machenschaften zu sein, bestärkt. Die Vorstellung von der „Ummah“ (der Gemeinschaft aller Muslime) beschränkt Freiräume für eine Bewusstseinsbildung, durch die individuelles Denken ermöglicht wird. Dieser

${ }^{7}$ Max Horkheimer/Theodor W. Adorno, Dialektik der Aufklärung. Philosophische Fragmente, Frankfurt a. M. 2008, S. 182.

8 ,Die Wohltat, dass der Markt nicht nach Geburt fragt, hat der Tauschende damit bezahlt, dass er seine von Geburt verliehenen Möglichkeiten von der Produktion der Waren, die man auf dem Markte kaufen kann, modellieren lässt.“ Ebd., S. 19.

${ }^{9}$ Ebd., S. 196.

10 Ebd.

11 Zur näheren Erläuterung des Ideologiebegriffs siehe Theodor W. Adorno, Beitrag zur Ideologienlehre, in: Ders., Gesammelte Schriften, Bd. 8, Frankfurt a. M. 1972, S. 457-477. - „Denn Ideologie ist Rechtfertigung. [Herv. i. O.] Sie erheischt ebenso Erfahrung eines bereits problematischen gesellschaftlichen Zustandes, den es zu verteidigen gilt, wie andererseits die Idee der Gerechtigkeit selbst, ohne die eine solche apologetische Notwendigkeit nicht bestünde, und die ihr Modell am Tausch von Vergleichbarem hat.“ Ebd., S. 465.

12 Horkheimer/Adorno, Dialektik der Aufklärung, S. 196. 
Kollektivismus erhält sich in Wechselwirkung mit der Aufrechterhaltung des Vollkommenheitsanspruchs des islamischen Sakralen. Das bedeutet aber nicht, dass der Islam außerhalb der Moderne steht, denn schließlich beansprucht heute das Kapitalverhältnis universelle Gültigkeit. Der Islamwissenschaftler Aziz Al-Azmeh schreibt in seinem 1993 veröffentlichten Buch „Islams and Modernities“ (deutscher Titel: „Islamisierung des Islam“), dass der Universalismus sich in den „Randzonen [...] wegen der Schwäche der ihm zugehörigen sozialen und kulturellen Bildungen unvollständig und ungleichmäßig“13 durchgesetzt hat. Diese Schwäche, so Al-Azmeh weiter, „findet ihr Gegenstück im Insistieren auf ,Authentizität"“.14

\section{Sehnsucht nach Authentizität und Wiedererweckungsbewegungen}

Letzteres zeichnet die erwähnte Sehnsucht nach der Konkretisierung des vermittelten Herrschaftsverhältnisses aus, ohne die Herrschaft aufzuheben. Die Forderung nach einer der Ummah entsprechenden Herrschaftsordnung - dem islamischen Kalifat - ist daher eine rückwärtsgewandte Utopie im Sinne der „Wiederherstellung einer Natur, die von der kontingenten Geschichte außer Kraft gesetzt war". ${ }^{15}$ Diese Forderung nach dem Kalifat funktioniert der Ideologie nach ähnlich den Wiedererweckungsbewegungen des arabischen Baathismus und des europäischen Faschismus. Im nicht nur von Islamisten offen geforderten Rückbesinnen auf den Islam als Urreligion, als das einzig Authentische, worin also ein vermeintlich volkstümliches und ursprüngliches Kollektiv - ähnlich der Volksgemeinschaft in der NS-Ideologie oder gegenwärtiger rechter Ideologien reaktiviert werden soll, wird eine ontologische Dichotomie geschaffen: Ein authentisches „,islamisches Selbst“ auf der einen Seite wird den Verfälschern der Geschichte, denen der „innere Wert“, also das Authentische, abhandengekommen sei, auf der anderen Seite gegenübergestellt. ${ }^{16}$ Der Islam ist daher gleichzeitig moderne und antimoderne Revolte: Modern ist er deshalb, weil er sich an Prämissen der Moderne entfaltet, um die Klassengesellschaft mit dem Ziel einer neuen Gesellschaft, der islamischen Ummah, aufzuheben. Antimodern ist er, weil er dieser Klassengesellschaft eine antiaufklärerische Bewegung utopischen Charakters entgegensetzt.

Die inhaltliche Bestimmung des Islam ändert sich daher auf Basis der gesellschaftlichen Ordnung. Es ist nicht möglich, von einem einheitlichen Islam im Sinne der Schriftenlehre auszugehen, da die bestimmenden islamischen Elemente je nach Ort und Kultur variieren. Im inhaltlichen Bestimmungsprozess des Islam allerdings werden die reaktionärsten Strömungen in der jeweiligen Kultur überhöht. Die Unmöglichkeit der

13 Aziz Al-Azmeh, Die Islamisierung des Islam. Imaginäre Welten einer politischen Theologie, Frankfurt a. M. 1996, S. 59 .

14 Ebd.

15 Ebd., S. 47.

16 Ebd. 
Herstellung einer einheitlichen Ordnung lässt sich anhand der Scharia, dem islamischen Recht, nachvollziehen. Bei der Scharia handelt es sich um keinen „Gesetzeskodex oder irgendwie Kodifizierbare[s]“, vielmehr stellt sie „willkürlich gesetzte Einzelexemplare“17 als ein Bündel an Rechts- und Sittenvorstellungen dar, deren Funktion als einheitsstiftende und -erhaltende Gesetzgebung damit obsolet wird. Als Sammelsurium an islamischen Handlungen und Aussprüchen ist ihre praktische Anwendung im Sinne eines allgemeinen Bezugspunkts daher gar nicht möglich. Die Forderung nach einer islamischen Ordnung läuft somit auf Willkürherrschaft hinaus, in der je nach realem politischen Kräfteverhältnis, einzig in Bezug auf den Namen Islam als dem Sakralen, eine allgemeine Verbindlichkeit hergestellt werden soll:

„Islam erweist sich demnach als ein selbstbezügliches Verhältnis, das mittels Übertragung funktioniert; jedes Element, auf das diese sakrale Qualität übertragen wird - der Schleier, verschiedene Vorschriften in Bezug auf Nahrungsverbote und Erbschaftsangelegenheiten [...] - verliert durch die Übertragung seine Neutralität und erhält einen besonderen Wert. Auf diese Weise wird jedes Element zu einem stellvertretenden Ausdruck für den Islam und steht voll und ganz für ihn ein." ${ }^{18}$

\section{Ungeglaubter Glaube}

Als ein selbstbezügliches System, dessen Anspruch nicht die Nachvollziehbarkeit im Sinne eines allgemeinen Reglements ist, worin sich, wenn auch nur formell, alle vertreten fühlen, braucht es daher nicht notwendigerweise eine Schriftenlehre, um als islamisch zu gelten. Der Islam ist in diesem Sinne ein ungeglaubter Glaube:

„Denn es ist diese Eigentümlichkeit alles Islamischen - dass es eine bloße nominalistische Setzung ist -, was dem symbolischen Zuschreibungsakt eine massive Bedeutung verleiht und was zugleich die ,Islamisierung von Dingen und Verhältnissen zu einer Handlung macht, die Privileg der zuschreibenden und sinnstiftenden Instanz bleibt. Das Repertoire, aus dem man auswählen kann, ist riesengroß; nur eine beschränkte Zahl an Elementen wird als Ausweis islamischer Rechtsgläubigkeit geltend gemacht - und die Entscheidung über diese Elemente ist ein politischer Akt." ${ }^{\text {"19 }}$

Das Verhandeln, was islamisch ist und was nicht, ist ein ewiger Prozess, der sich aber nicht als politische Verständigung, sondern als Aneinanderreihung politischer Akte vollzieht. Im Gegensatz zur Hervorbringung eines persönlichen Glaubens an Gott, in dessen Rahmen Subjektbildung noch möglich ist, bestimmt sich der Islam im Äußeren, dem Kollektiv. Innerhalb der islamischen Ideologie werden daher keine Formen von Subjektwerdung, in denen das Ich Hauptmovens jeden Handelns ist, ermöglicht. Die

${ }^{17}$ Ebd., S. 44-45.

18 Ebd.

19 Ebd., S. 45-46. 
Anerkennung von Eigenverantwortlichkeit wird daher nicht eingeräumt. Der Islam schafft somit tendenziell keine Verhältnisse, in denen Konflikte bewusst gemacht werden können. Man fühlt sich stets als Objekt und nicht als Subjekt. Damit führt jede Kränkung, jede Triebversagung, die man sich selbst auferlegt oder durch das islamische Kollektiv auferlegt bekommt, weder zu einer Auseinandersetzung und Hinterfragung seiner selbst noch zu einer Konfrontation mit den rigiden Werten innerhalb des Islam, sondern wird den Kafir zugeschrieben. Durch den islamischen „Zuschreibungsakt“ entsteht eine Dynamik, in der diejenigen Kräfte die Oberhand gewinnen, die sich auf die restriktivsten Elemente im Islam beziehen. Jeder, der sich auf diesen Allgemeinbegriff Islam bezieht und die politische Durchsetzungskraft hat, kann sich Moslem nennen. So setzte sich beispielsweise der damalige irakische Diktator Saddam Hussein, der selbst der pro forma säkularen, arabisch-faschistoiden Baath-Partei angehörte, in den 1990er-Jahren als Führer einer islamischen Gemeinschaft in Szene, um seine Herrschaft zu festigen. ${ }^{20}$ Seine Reislamisierungs-Kampagne erst hat die politische Voraussetzung für das Entstehen des sogenannten Islamischen Staats geschaffen. ${ }^{21}$

Genauso wenig, wie der Islam Einheit stiften kann, schafft er ein homogenes islamisches Kollektiv. Dies wird an der Barbarei des sogenannten Islamischen Staats am deutlichsten, insofern seine Mitglieder auch Muslime ermordeten, um sich ihre „Authentizität“ selbst zu beweisen. Es ist zwar nicht möglich, sich mit dem spontan wandelbaren Gebilde, dem Resultat dieser „nominalistischen Setzung“, als Einheit zu identifizieren, aber der Name Islam als Abgrenzung nach außen täuscht die Homogenität nach innen vor. Die Diffusion bei gleichzeitigem Anschein von Homogenität findet sich am radikalsten beim IS, ist aber bereits an der Vielzahl von Imamen zu sehen, die bei Fernsehdebatten darüber diskutieren, was islamisch verpönt ist und was nicht. Dabei bestimmt sich, wie erwähnt, in den einzelnen Aussagen selbst der islamische Glaubensinhalt immer wieder aufs Neue. Die innerislamischen Revolten sind daher eine Konsequenz dieser Ideologie und werden auch bei jungen Jihadisten offensichtlich. In ihrem Hass auf die westliche Gesellschaft begehren sie zuallererst gegen den Islam ihrer Eltern auf, weil ihnen dieser nicht islamisch genug ist. Bei diesem Aufbegehren geht es nicht um ein Brechen mit den elterlich vermittelten Werten, sondern um deren Überhöhung. Der Jihadismus ist keine Abspaltung des Islamismus vom Islam, sondern seine konformistische Aneignung. Daher ist eine klare Trennung zwischen Islam und Islamismus nicht möglich.

Der Bezug zum Islam von jungen Muslimen, die in Europa aufwachsen - also in Staaten, die zumindest formell säkular sind - ist anders bestimmt. Der islamische Wahrheitsanspruch, der innerhalb der islamischen Communities in Europa im Privaten reproduziert wird und der auch immer wieder in die politische Öffentlichkeit drängt,

20 Siehe Michael Weiss/Hassan Hassan, ISIS. Inside the Army of Terror, New York 2015, S. 23. - So wurde der irakischen Flagge, auf der zuvor die drei Sterne, welche den Baathismus mit den Schlagworten Einheit, Freiheit, Sozialismus symbolisieren sollten, „Gott ist groß“ hinzugefügt. Auch wurde vermehrt das Scharia-Recht angewendet. 21 Kyle W. Orton, How Saddam Hussein Gave Us ISIS, in: The New York Times, 23.12.2015, [https://www.nytimes.com/2015/12/23/opinion/how-saddam-hussein-gave-us-isis.html], eingesehen 9.11.2018; 
stößt in einer säkularen Gesellschaft bereits im Alltag an seine Grenzen. Damit ist der Islam im Sinne der erwähnten „,nominalistischen Setzung“ als Abgrenzungsmerkmal von der Mehrheitsgesellschaft bestimmt. Da es sich beim Islam um einen ungeglaubten Glauben handelt, muss die religiöse Identität nach außen umso präsenter auftreten: Um den Glauben für sich glaubhafter zu machen, muss umso mehr die Richtigkeit der rigiden Moral- und Sittenvorstellungen nach außen zur Schau gestellt werden. Der belgische Polizist Hamid Benichou bringt dieses Paradoxon auf den Punkt: „,Warum sollte ich mir auf die Fahne schreiben ,Schaut her, ich bin Moslem. [...] Heißt das ich bin nicht sicher, ob ich Moslem bin? Dass meine Eltern keine Moslems waren?" 22

Die Radikalisierung wird im Jihadismus am offensichtlichsten, aber diese Tendenz ist bereits bei jenen Migrantinnen der zweiten und dritten Generation zu sehen, die sich für die Verschleierung entscheiden. Der Islam ist hier politisches Statement der bewussten Abgrenzung von und Auflehnung gegen die als feindlich erklärte säkulare Gesellschaft allerdings auf Basis eben dieser Gesellschaft. Die Politologin Seyla Benhabib schreibt über Schülerinnen in Frankreich, die sich weigerten das Kopftuch abzulegen und damit eine Verschleierungs-Debatte auslösten, dass „es gerade die egalitären Normen des öffentlichen französischen Bildungssystems" waren, die den Mädchen das Selbstvertrauen gegeben haben, aus den „häuslich patriarchalen Strukturen“ auszutreten und öffentlich für ihr Recht auf freie Religionsausübung einzustehen. ${ }^{23}$ Obwohl laut dem Koran keine Pflicht zur Verschleierung besteht und es durchaus viele Frauen gibt, die kein Kopftuch tragen und sich trotzdem als Muslimas verstehen, deuteten diese jungen Mädchen das Tragen des Kopftuchs als ein Symbol des Protests gegen den säkularen Staat um.

Da sich die islamische Qualität durch politische (Abgrenzungs-)Akte bestimmt, führt jedes staatlich gesetzte Verbot zu einer weiteren Politisierung. ${ }^{24}$ Oft werden in Reaktion auf Gesetze, welche die Religionsfreiheit einschränken, von Islamverteidigern Partikularrechte gefordert, mit deren Gewährung der Staat seine Souveränität aufgeben würde. Nicht zufällig beziehen sich daher Islamkritiker auf ein einheitliches Gesetz und damit für staatlich gesetzte (Verschleierungs-)Verbote. Sie sind in ihren Anliegen nicht, wie ihnen oft von linker Seite vorgeworfen wird, gegen den Pluralismus in der Gesellschaft oder gar antidemokratisch, sondern wollen die Basis, auf der dieser Pluralismus gedeiht, also den bürgerlichen Staat, geschützt wissen.

22 Thomas Lauterbach, Europas Muslime. Auf Reisen mit Nazan Gökdemir und Hamed Abdel-Samad. Teil 2: Belgien, Frankreich und Spanien, ARTE/ZDF, $52 \mathrm{~min}$, 2017, 4:12-4:24 min., [https://youtu.be/97cRnJ26zds?t=4m12s], eingesehen 13.11.2018.

${ }^{23}$ Seyla Benhabib, Kosmopolitismus ohne Illusionen. Menschenrechte in unruhigen Zeiten, Berlin 2016, S. 248.

24 Ebd., S. $247 f$. 


\section{Universalisierte Opferschaft}

Gleichzeitig ist es allerdings auch dieser bürgerliche Staat, der seinen ideologischen Reflex, die modern-antimoderne Revolte hervorbringt. Die islamische Ideologie steht daher im Einklang mit all jenen Strömungen, die ebenfalls von einer grundlegenden Staats- und Kapitalismuskritik nichts wissen wollen. Ihre Wirkmächtigkeit liegt darin, dass sie erfolgreich die reaktionären Kräfte im allgemeinen Herrschenden anspricht. Die islamische Ideologie muss daher ähnlich wie rechte Ideologien begriffen werden. Der Anspruch auf Authentizität, also das Wieder-Natur-Werden in der unmittelbaren Übereinstimmung von Gesellschaft, Staatswesen und Kultur in der Gemeinschaft ist ebenso im rechten Gedankengut zu finden. Es ist daher kein Zufall, dass es Sympathien für den Islam seitens Rechtskonservativer gibt, auch wenn das heutzutage in Europa nur mehr selten offen propagiert wird. Beispielsweise bezeichnete Gabor Vona, der Chef der rechtsextremen ungarischen Jobbik, den Islam als „letzte Hoffnung des Konservativismus". 25

Sowohl den rechten als auch den linken Islamverteidigern ist gemein, dass sie in ihrer „Apologie des Anderssein“ 26 dem Islam und damit „den“ Muslimen ein unveränderliches Wesen attestieren. Rechte erkennen das Mobilisierungspotential des Islam und beneiden, sofern sie nicht sogar konvertieren, aus der Ferne jene islamischen Staaten, die ohne die Gebundenheit an demokratische Instanzen frei gegen Juden, Andersgläubige, Frauen und Homosexuelle hetzen. Sofern sie sich gegen den Islam aussprechen, dann nur als Konkurrenten gegenüber einer Ideologie, die erfolgreicher in der Bindung reaktionärer Kräfte ist. Dieses Mobilisierungspotential insgeheim beneidend, wird der eigene Antisemitismus externalisiert und „den“ Muslimen zugeschrieben. Sie sind daher keine Islamkritiker, sondern wollen sich eines Konkurrenten - im „Kampf“ um das für das eigene Kollektiv beanspruchte Herrschaftsgebiet in Form des Nationalstaats entledigen. Es ist die fatale Konsequenz dieses Konkurrenzverhältnisses, dass Menschen (unabhängig davon, wie diese es mit dem Islam halten), die auf der Flucht nationalstaatliche Grenzen überwinden müssen und damit ohnehin das Ressentiment völkischer Nationalisten auf sich ziehen, zu Muslimen gemacht werden.

Der Erfolg der islamischen Ideologie in Europa rührt aber nicht ausschließlich aus dem Konkurrenzverhältnis der Rechten zum Islam, denn auch linke bzw. sich als progressiv Verstehende brauchen die Konservierung der ,authentischen“ Kultur. Genauso wie auf der rechten Seite ist bei den Linken in Bezug auf den Islam eine „Ehrfurchtshaltung gegenüber dem Archaismus“ $27 \mathrm{zu}$ finden. Im Kultivieren der hiermit verbundenen Differenz werden universalistische Werte wie Gleichheit und Freiheit aufgegeben. Dennoch bezieht sich auch die Linke in der Verteidigung antidemokratischer

${ }_{25}$ Die Presse, 11.2.2016, S. 4.

26 Al-Azmeh, Islamisierung des Islam, S. 38.

${ }^{27}$ Ebd., S. 8. 
Strömungen und reaktionärer islamischer Werte oftmals auf die Menschenrechte und verkehrt den zugrundeliegenden Universalismus damit in sein Gegenteil.

Das heutige Verständnis von Menschenrechten in Europa wiederum entwickelte sich als scheinbare Lehre aus Nationalsozialismus und Holocaust. Dabei wurde der Holocaust örtlich, zeitlich und schlussendlich historisch entkontextualisert. Die Konsequenz war die Verwischung der Grenzen zwischen Tätern und Opfern:

„Der Preis der Entkontextualisierung führt auch dazu, dass es in diesem Diskurs keine Juden und keine Deutschen mehr geben darf. Es gibt nur Menschen und Menschheit, wie auch aus dem Begriff der, Verbrechen gegen die Menschheit' und der Entstehung eines moralischen und legalen Kodex gegen ,Völkermord' hervorgeht." 28

Das gemeinsame Maß für das europäische Projekt bildete daher eine unterschiedslose Opferschaft und der Holocaust wurde „zum Maßstab des eigenen Leidens stilisiert““ ${ }^{29}$ Mit der Universalisierung der Holocaust-Erinnerung wurde auch die Bedeutung des jüdischen Opfers universalisiert, wobei es nicht um eine Auseinandersetzung mit den nationalsozialistischen Verbrechen - das heißt dem zur Tat gebrachten Vernichtungsantisemitismus - ging. Somit war es nun ,vielen Opfergruppen erlaubt, sich in den jüdischen Opfern wiederzuerkennen“. ${ }^{30}$ Diese Auflösung von Geschichte drückt sich im Menschenrechtsdiskurs ${ }^{31}$ aus, in dem das Menschsein a-priori Opfer-Sein bedeutet. Im europäischen Gedächtnis ,geht es in erster Linie um Verfolgung, Demütigung, Hass. Jeder wird zum Opfer".32 Im Verteidigen des Islam seitens sich als progressiv Verstehender wurden zuerst die Palästinenser und später verallgemeinert die Muslime als Opferkollektiv gesetzt, was allzu gern von islamischen Verbänden in Europa übernommen wird. Immer öfter wird auch unter folgendem Slogan mobilisiert: „Du musst kein Moslem sein, um für Gaza einzustehen. Es reicht, ein Mensch zu sein“.33

Dabei darf nicht vergessen werden, dass in Europa in der Vergangenheit islamische Verbände mehr Anerkennung und Unterstützung durch die Staatsgewalt erfuhren als beispielsweise migrantische Interessensverbände, die sich für eine soziale und materielle Besserstellung einsetzten. Der in den europäischen Staaten unterschiedlich vorangetriebene Multikulturalismus bestärkt islamische Vereinigungen und ignoriert Säkulare und Ex-Muslime. In Wien organisierte eine dieser islamischen Vereinigungen, die AKPnahe Union Europäisch-Türkischer Demokraten, im Sommer 2014 eine Free-Gaza-

\footnotetext{
${ }^{28}$ Daniel Levy/Natan Sznaider, Erinnerung im globalen Zeitalter: Der Holocaust, Frankfurt a. M. 2007, S. $12 \mathrm{f}$.

${ }^{29}$ Ebd., S. 92.

${ }^{30}$ Ebd., S. 62.

31 „Wenn man verstanden hat, dass der Menschenrechtsdiskurs ein entkontextualisierter Diskurs ist - da er sich der historischen Einbettung von Menschen verweigern muss, sonst wäre es ja kein Menschenrechtsdiskurs, sondern ein Bürgerrechtsdiskurs -, dann wird man auch verstehen, warum der Holocaust als symbolische Repräsentation des Bösen entkontextualisiert wird.“ (Ebd., S. 15.)

32 Ebd., S.10.

33 Viele der Pro-Gaza-Demonstrationen verliefen unter diesem Slogan. Siehe exemplarisch eine Demonstration in der Frankfurter Innenstadt im Juli 2014: Peter Jülich, „Du musst kein Moslem sein um für Gaza einzustehen“, 13.7.2014, [https://peter-juelich.photoshelter.com/image/I000080fJVCiMgZA], eingesehen 13.11.2018.
} 
Demonstration, bei der unter anderem auch Fahnen der Terrororganisation Hamas zu sehen waren. ${ }^{34}$ Einen Tag zuvor hatte der damalige türkische Premierminister Recep Tayyip Erdogan, der sich zu dieser Zeit als Kandidat im Rennen um die türkische Präsidentschaft befand, bei einer Wahlkampfveranstaltung Israel ,barbarischer als Hitler" genannt. ${ }^{35}$ Er wusste, dass er auch die Muslime in Europa damit mobilisieren konnte. In der durch islamische Agitatoren vorangetriebenen Universalisierung des Opferstatus soll sich jeder mit „den“ Muslimen als entrechteten, betrogenen Menschen identifizieren, um schlussendlich gegen Israel vorzugehen. Leo Löwenthal, der in den 1940er-Jahren eine Untersuchung über Agitationsmechanismen durchgeführt hat, charakterisiert den Agitator folgendermaßen:

„Er steigert die Stimmung von Misstrauen bei seinen Hörern, indem er diese daran erinnert, dass sie ,einer Fremdherrschaft unterworfen' und dass sie ,ständig von schlimmen Machenschaften bedroht ${ }^{\star}$ seien. Sie alle, werden am laufenden Band betrogen': bei der Rationierung, im Krieg, durch die Presse und das Kino. Solch skrupellosen Taktiken steht, das schlichte, gewöhnliche, redliche Herdenvolk' hilflos gegenüber; es ist immer das Opfer, das ewig hereingelegte." 36

Das Heraufbeschwören dieser „unbewussten Mechanismen“37 zeichnet solche Agitatoren wie Recep Tayyip Erdogan und seine Vertreter in Europa aus. Sie verlassen sich auf das reproduzierte Opfer-Gefühl, wie man nicht nur an den Pro-GazaDemonstrationen im Sommer 2014, sondern auch den jährlich stattfindenden Al-Quds Aufmärschen sehen kann. Multikulturalismus-Verteidiger des Islam leugnen daher nicht das „grundlegende Opfergefühl europäischer Muslime“"38 oder die ,globale[n] muslimische[en] Opferrolle“"39, da sie die Muslime in dieser Opferrolle bestätigt wissen wollen. Sie selbst gehen in dieser Opferrolle auf und benutzen das klassenübergreifende islamische (Opfer-)Kollektiv, im Sinne eines antisemitischen Missbrauchs der Menschenrechte gegen „die“ Juden.

\section{Schlussbemerkung}

Der islamische Antisemitismus, dessen Erfolg in Europa spätestens seit den Pro-GazaDemonstrationen im Sommer 2014 offensichtlich ist, ist daher nicht nur eine Konsequenz der politischen Kräfteverhältnisse in den islamischen Ländern, sondern muss auch konkret auf den Diskurs der Linken und Rechten über den Islam sowie die

34 Teresa Eder/Jutta Berger, Tausende in Wien und Bregenz bei Demos gegen Israels Militäreinsatz, in: Der Standard, 20.4.2014, [https://derstandard.at/2000003312679/Israel-Proteste-in-Vorarlberg], eingesehen 13.11.2018.

35 Die Presse, 21.7.2014, S. 2.

${ }^{36}$ Leo Löwenthal, Falsche Propheten. Studien zum Autoritarismus (Schriften, Bd. 3), Frankfurt a. M. 1990, S. 36.

${ }^{37}$ Ebd., S. 12.

38 Timothy Garton Ash, Der Islam in Europa, in: Thierry Chervel/Anja Seeliger (Hrsg.), Islam in Europa. Eine internationale Debatte. Frankfurt a. M. 2007, S. 30-54, hier S. 34.

${ }^{39}$ Ebd., S. 42. 
Zugeständnisse der jeweiligen europäischen Regierungen an islamische Vereinigungen zurückgeführt werden.

Kritisiert man den Islam, unabhängig davon wie differenziert diese Kritik auch ist, wird häufig der Vorwurf der „Islamophobie“ erhoben. Die Verwendung des Begriffs „Islamophobie“, als vermeintlich antirassistisches Argument, kehrt jedoch die berechtigte Angst unter den Tisch, die viele Menschen, die in islamischen Ländern und Communities aufgewachsen sind und aus diesen fliehen, vor dem Islam haben. Das Appeasement der nur scheinbar Links-Progressiven überlässt diese Menschen, wie überhaupt die Kritik am Islam den Rechten, die das Thema für ihre Agitation gern aufgreifen. Eine progressive Linke sollte begreifen, dass es im Gegensatz zur rechten „Islamkritik“ auch eine emanzipatorische Islamkritik gibt, die nicht als antimuslimischer Rassismus abgetan werden kann. Essenzialismus von links, der eine indirekte Ungleichbehandlung fordert, ist mit dem Universalismus selbst entgegenzuhalten, dass überall Verhältnisse geschaffen werden müssen, die es ermöglichen, zum mündigen Denken zu gelangen. Ein Ansatz wäre, säkulare Kräfte und Initiativen kritisch-politischer Bildungsarbeit nicht nur in Europa zu fördern, sondern diese darüber hinaus auch in islamischen Ländern zu unterstützen. Dabei geht es nicht darum, dass man Menschen irgendeinen europäischen Wertekanon aufzwingt, sondern darum aufzuzeigen, dass das „Sapere Aude!“, das Kant'sche Diktum „Habe Mut, dich deines eigenen Verstandes zu bedienen“, jedem zumutbar ist. Das wäre die emanzipatorische Wendung des Universalismus. 
